\title{
QUALITY STRUCTURING OF MEDICAL SERVICES BY DECOMPOSITION OF QUALITY FUNCTION
}

\author{
Mykola Mykyichuk, Dr.Sc., Prof. \\ Lviv Polytechnic National University,Ukraine; e-mail:mykolamm@ukr.net \\ Olesia Chaban, Ph.D., As.-Prof. \\ Lviv National Medical University named after Danylo Halytsky, Ukraine
}

\begin{abstract}
Quality medical care is an important indicator of the economic development of a country. Today, improving the quality of health care services is a priority for both health care institutions and the state as a whole. The development of methods and tools for continuous improvement of the quality of medical services promotes regulation, the perfection of those services, ensures the effective working of all the components together and the consistency and continuity of their provision, as well as help the staff to determine how to achieve the optimal level of care. The implementation of modern methods of ensuring the quality of products and services in medical practice is aimed at increasing patient satisfaction and receiving adequate and professional medical care, in accordance with the principles of Good Clinical Practice. Therefore, it is important to summarize and systematize the requirements for healthcare delivery processes. A key element of the quality management model of care is a process analysis, which involves the identification and design of processes along with their continual improvement. At the same time, the result of management is based on a cycle of actions aimed at minimizing the frequency of deviations in key processes, which guarantees the achievement of the best potential scientifically verified results. There are several groups of processes in a medical institution: treatment, diagnostics, supply, etc. Defining the function of the quality of medical services and their decomposition will allow for optimizing the work of medical institutions and encourage efficiency and competitiveness in innovative development. The article presents the scheme of optimization of medical services by decomposition of their function of quality. A graphic model of decomposition of the function of the quality of health services is proposed based on the model of relations which will formalize the requirements for the processes of providing medical services in a medical institution. This will help to provide healthcare services gradually and minimize the loss of quality of the provided medical services.
\end{abstract}

\section{Keywords}

Medical services, Process of optimization, Mathematical model, Quality function

\section{Introduction}

Quality medical care is an important indicator of economic development in the country. Today, improving the quality of health care services is a priority for both healthcare facilities and the state as a whole. The implementation of modern methods of ensuring the quality of products and services in medical practice is aimed at increasing patient satisfaction and receiving adequate and professional medical care, in accordance with the principles of best medical practice, so it is important to generalize and systematize the requirements for the processes of health care delivery [1]. Defining the function of the quality of medical services and its decomposition will allow optimizing the work of medical institutions and ensuring their efficiency and competitiveness in the conditions of innovative development.

\section{The current state of the problem}

In the area of process management, the main and ancillary processes are distinguished. The primary process at a healthcare facility is the process of providing health care. Medical service, in turn, involves a set of various processes, the effectiveness of management of which is determined by the diversity of indicators. Therefore, the level of quality of health care services can be enhanced by the manageability and consistency of three key elements: the structure, process, and result of treatment. An important task of ensuring the required quality of services is structuring health care services and regularly monitoring their compliance with the standards of structure and process in a healthcare facility. Therefore, the systematization of the requirements for the medical service and the creation of methods for ensuring its quality is an urgent task to improve the efficiency of the medical institution.

\section{The goal of the work}

The purpose of this work is to develop an approach for decomposition of the quality function of medical services in medical institutions. 


\section{Statement of the main material}

In the healthcare delivery process, the quality reflects the degree of appropriateness of the cooperation of medical staff, patient and the effectiveness of this cooperation. The main processes in a healthcare institution are the medical care and the health care delivery processes. The basic processes are unique, their results are related to a specific patient with a specific clinical condition and nosological form, and the results of assisting processes are used by many functional and structural units of the healthcare institution. Auxiliary processes include processes of resource management, management activity, monitoring, and etc. [2].

All activities of the medical organization, aimed at ensuring high quality of diagnostics and treatment, should be incorporated in a logical chain that allows us, successfully developing the results achieved in the previous stage, to ensure the heredity of the patient's monitoring data and the positive dynamics of the treatment of the disease. Assessing the quality of care is one of the criteria for the effectiveness of the implementation of various innovations in the activities of medical institutions, which in turn increases the efficiency of medical services. The efficiency of medical services reflects the degree of achievement of specific final results at certain labor and material costs, fulfillment of the tasks. The effectiveness of the industrial model of healthcare institution management is closely linked to increased clinical performance while minimizing the cost of health care services and their accessibility to patients [3].

Medical institutions (MI's) are complex organizational systems with many interconnected processes the quality of which is determined by a large number of indicators [4]. Therefore, having a quality management system in a medical institution and applying the basic principles of continuous improvement of the quality of services determines to a great extent the controllability of the basic processes and, consequently, the degree of compliance of the medical services with the established requirements. In addition, the systematization of requirements for the processes of the provision of medical services (MSs) and the creation of methods for ensuring their quality are the basis for improving the efficiency of the medical institution. The issue of improving the efficiency of the MI's work is urgent, however, by now, there is no definite solution. This is mainly due to the complexity of the processes of providing medical services, which contain a significant number of regulatory, organizational, technical and scientific-methodological factors. Measuring and controlling the quality of care is an informative basis for health care delivery processes, and serves to produce the timely and credible information needed to manage health care delivery processes.

Therefore, only quality control indicators of health care cannot be used to assess the quality of health care services, since they actually only characterize the outcome. Important elements, quality assessment of which is used to choose effective ways of managing the processes of the provision of medical services, assessment, and forecasting of compliance of medical services, solving other tasks of the MI's, are left out. In the first stage of structuring the indicators of quality of medical services, it is advisable to carry out their rational minimization.

For quantitative evaluation of the quality of MS's, it is necessary to choose algorithms and indicators, taking into account the relevant requirements, principles, and rules important in ensuring the quality of products and services.

Let us consider the MS system $-S_{M S}$ as a global process [5], which combines $n+1$ local sub-processes $S_{M S}, 1 \leq i \leq \mathrm{n}$, each with a $x_{i}$ input and a $y_{i}$ output. Given the variety of possibilities for building an MS system, the outputs of one of its subsystems may be the inputs of other subsystems or links with the metasystem. It is reasonable to consider [6] that there is some function - $G_{M S}$ which we will call the quality function of the MS system, which we will use to evaluate the process of MS system functioning. Let us consider that the purpose of the MS system operation is to maximize the quality function $G_{M S}$ by effectively coordinating the multiple elements $G_{i}$ of the MS system and the set of relationships $M_{S}$ between the elements of the MS system.

The condition of maximization of quality function $G_{M S}$ can be achieved by making optimal use of the available elements and links, which is equivalent, from the point of view of system analysis [6,7] to making effective management decisions that can be represented in the form of a multitude of solutions for the MS system:

$$
D_{M S}=\left\{D_{1}, D_{2}, \ldots D_{i}, \ldots D_{n+1}\right\} \rightarrow o p t
$$

By choosing from a variety of possible $D_{M S}$ solutions, the optimal ones can maximize the quality function $G_{M S}$ of the MS system.

A typical structure of an MS system can be represented as a three-level hierarchical system [5], where the first level is the MS system - $G_{M S}$, the second level is the service quality assurance subsystem - $G_{Q}$ and service efficiency subsystem $-G_{E}$, and the third level includes the elements: $G_{S P}$ - standardization of diagnostic and therapeutic processes; $G_{V S}$ - validity of medical services; $G_{A A}$ - the adequacy of the amount of medical services and diagnostics; $G_{L C}$ - the level of competence of the medical staff; $G_{C A}$ - consistency of actions between participants in the process; $G_{C T}$ - availability of modern clinical technologies; $G_{M Q}$ - monitoring the quality of health services provided; $G_{M B}$ - 
availability of a database of normative documents of medical care and services. This approach provides additive properties of the system allowing linear convolution (unfolding) of performance indicators from the lower levels to the upper level [8].

Let us present a graphical model of the MS system in the form of a model (Figure 1), which allows us to carry out the decomposition of functions of elements with hierarchical levels and to provide reasonable analysis and establishment of rational values and relations between quality indicators of individual elements of MS.

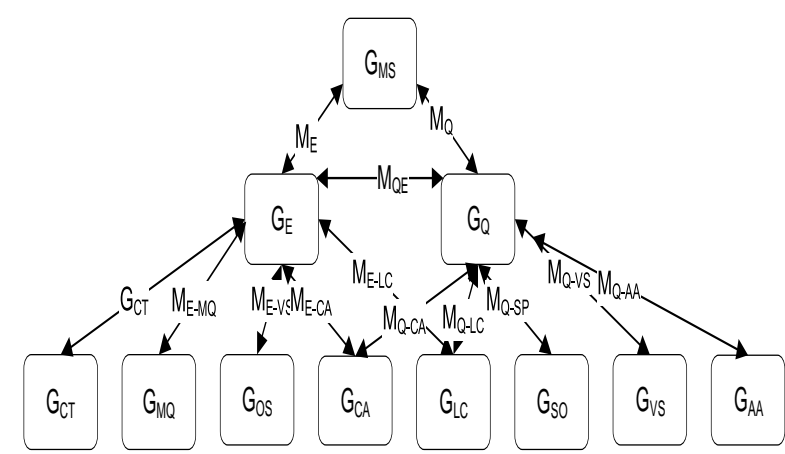

Fig. 1. Graphic model of decomposition of the quality function of the health care system

The condition for maximizing the quality function $G_{M S}$ by making effective control decisions (1) can be represented by the following formula, which also reflects the effect of the quality functions of subsystems and elements $G_{i}$ on the quality function $G_{M S}$ :

$$
G_{M S} \stackrel{D \rightarrow o p t}{\longrightarrow} \sum_{i=1}^{n} M_{i} \cdot G_{i}=\sum_{i=1}^{n} M_{i} \cdot \sum_{j=1}^{m} M_{i j} G_{i j},
$$

where $M_{i}$ - is the link weight of the ith subsystem $G_{i}$ is $G_{M S} ; M_{i j}$ - multiple (weight) connections of the $j^{\text {th }}$ element to $i^{\text {th }}$ subsystem $G_{i}$. The decomposition of MS system quality functions is feasible considering that the efficiency of MS system elements is primarily determined by the efficiency of conversion of $\mathrm{x}_{i j}$ inputs (goals) to $\mathrm{y}_{i j}$ outputs, which can reflect dependencies (3):

$$
G_{i j}=u_{i j} \frac{x_{i j}}{y_{i j}} \stackrel{d_{o p t}}{\longrightarrow} \max
$$

here $u_{i j}$ are the effects of converting $x_{i j}$ inputs to $y_{i j}$ outputs that determine the quality of performing their function by a separate MS element. For real MS systems, it is difficult to uniquely determine the effect of converting $x_{i j}$ inputs to $y_{i j}$ outputs [9]. Decision-making presupposes a certain goal to which the decision is directed. The existence of a decision problem indicates that not all solutions in multiple $D$ achieve the goal. Let 's split set $D$ into three subsets [9]:

$$
D=D^{+} \cup D^{o} \cup D^{-}
$$

here $D^{+}$- "good solutions," those that approach the goal; $D^{-}{ }_{-}$"bad decisions," those that remote from the goal; $D^{0}$ - "neutral decisions", that is, those that do not affect the achievement of the goal.

Obviously, the optimal $d_{\text {opt }}$ solution must belong to a set of good solutions: $d_{\text {opt }} \in D^{+}$. The decision task requires a decision criterion $Q$ to exist. In the current state of medical institutions, which determines the satisfaction of patients 'needs and ensuring the competitiveness of the medical institution, the vector of optimal solutions should take into account the vectors of the service provider 's priorities - $W_{l}$ and those of a consumer - $W_{2}$ :

$$
d_{o p t} \in D_{W 1}^{+} \cap D_{W 2}^{+}
$$

Now vectors of priorities of service provider - $W_{l}$ and the consumer - $W_{2}$ are established in the form of standardization of risks of service provider - $\alpha$ and the consumer - $\beta$, which in the conditions of lack of the formalized methods of establishment of communication of these risks with the risk of loss of quality of services creates additional difficulties for expeditious making of optimal decisions. 
Therefore, for complex organizational and technical systems such as MI, optimal decision-making cannot be a single action but must be carried out periodically taking into account changes in its operating conditions. An important task of maximizing the quality function is to create a method of improvement by performing the iterative procedures, which will allow optimizing the efficiency of the MS system while meeting the conditions of minimization of risks of the consumer and the service provider. In carrying out such a task, the complexity of decision-making lies in the impossibility of explicitly taking into account subjective influences on the effectiveness of these decisions, which requires the search for models of formalization of requirements to the MS system.

To formalize the MS system requirements, we present below a generalized image of the MS system element as a relationship model (Figure 2).

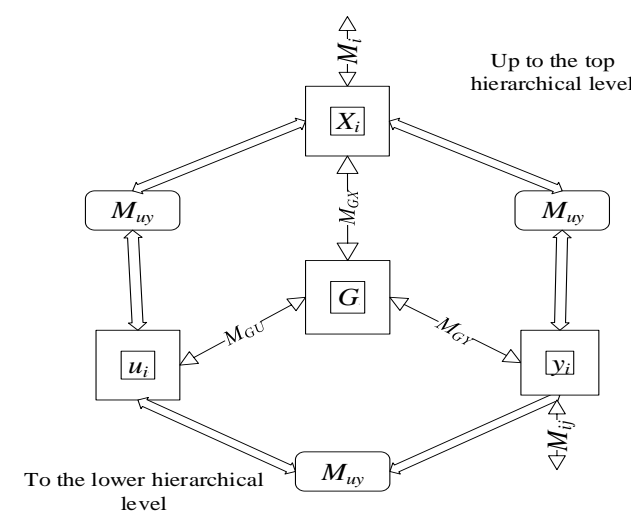

Fig. 2. Graphic model of relations of the $i^{\text {th }}$ element of medical services

Then, taking into account the relationship model (Figure 2), the quality function $G_{i}$ of the $i^{\text {th }}$ element is represented as:

$$
G_{i}=\left|M_{U X}, M_{G U}, M_{G Y}\right| u_{i} \frac{x_{i} \cdot\left|M_{U X}, M_{G X}, M_{Y X}\right|}{y_{i} \cdot\left|M_{U Y}, M_{G Y}, M_{Y X}\right|}
$$

For complex systems, it is difficult to explicitly take into account the influence of $M_{i j}$ relationships among $x_{i j}, y_{i j}, u_{i j}$, so when building real systems, it is necessary to minimize the influence of secondary links by ensuring that the following conditions are met:

$$
\begin{aligned}
& M_{U X}, M_{G X}, M_{G Y}, M_{U Y} \Rightarrow 0 \\
& M_{G U}, M_{Y X} \Rightarrow s e t
\end{aligned}
$$

Then the expression (6) can be simplified to the following form:

$$
G_{i}=M_{G U} \cdot u_{i} \frac{x_{i}}{y_{i}}+M_{Y X} u_{i} \frac{x_{i}}{y_{i}} \approx M_{G U} \cdot u_{i} \frac{x_{i}}{y_{i}}+\varepsilon_{G I},
$$

where $\varepsilon_{G I}$ is the inaccuracy of the evaluation of the quality function $G_{i}$ of the $i^{\text {th }}$ element. Given formula (3), it can be considered that the quality function $G_{i}$ of the $i^{\text {th }}$ element depends on the efficiency of converting inputs $x_{i}$ to $y_{i}$ outputs, herewith the efficiency is determined by the corresponding $u_{i}$.

Mostly, determining the conversion effects of $u_{i}$ elements of MS system is a complex task that is difficult to reflect as a functional dependency, so one way to solve this problem is to use logic-mathematical modeling, which will increase the formalization of the MS improvement process. Since the transformation effect $u_{i}$ inherently determines the degree of correlation between $x_{i}$ and $y_{i}$, it is logical to express it as a correlation coefficient $r_{i}$, allowing for extensive use of correlation analysis techniques. For the MS system model shown in Figure 1, the expression for the quality function, taking into account (2), (3), (8), will look as follows: 


$$
\begin{aligned}
& G_{M S} \stackrel{D \rightarrow o p t}{\longrightarrow} M_{E} \times G_{E} \cap M_{Q} \times G_{Q}+\varepsilon_{M S}, \\
& G_{E}=\left\|M_{E-i}\right\|^{i=\overline{1, n}} \times\left\|u_{i j}^{E}\right\|^{i=\overline{1, n}}, \\
& G_{Q}=\left\|M_{Q-i, m}\right\|^{i=\overline{1, n}} \times\left\|u_{i j}^{Q}\right\|^{i=\overline{1, n}},
\end{aligned}
$$

Formalizing the tasks of evaluating the effects of individual elements of the MS system, as well as the inaccuracy of modeling its quality function, seems to be a quite complex scientific problem that requires separate research. Currently, the method of structuring quality function can be used to evaluate the effectiveness of MS and its individual elements, and the assessment of inaccuracy of MS quality function mapping by the model (9) can be estimated from the results of successive studies of its compliance with efficiency indicators.

\section{Conclusions}

The analysis shows that in order to increase the efficiency of the medical services system, it becomes important to minimize the impact of subjective and methodological factors on the quality of medical services. Since the negative influence of subjective factors is determined by the low level of qualification of personnel and the organization of medical services, the main way of their minimizations seems to be the systematization of the requirements for these services on the basis of the proposed hierarchical system.

The systematization of the requirements for the health-care system should be carried out by defining a model of its quality function and ensuring conditions for the optimization of its individual processes and connections between them. It is advisable to optimize the structure and connections of the elements of the medical services system with the help of the proposed model of relations.

The approach to the decomposition of the quality function would formalize the process of establishing requirements for the elements of the medical services system and contribute to minimizing the risks of loss of services quality.

\section{Conflict of Interest}

The authors claim that there are no possible financial or other conflicts over the work.

\section{References}

[1] A1-Abri, Rashid, Amina A1-Balushi, "Patient satisfaction survey as a tool towards quality improvement", Oman medical journal Vo1.29.1, pp. 3-7, 2014.

[2] A. Donabedian, "Evaluating the quality of medical care", Milbank. Mem. Fund. vol. 44. pp. 153-166, 1996.

[3] C. Borrill, J. Carletta, A. Carter, J. Dawson, S. Garrod, The Effectiveness of Health Care Teams in the National Health Service, DOH Final Report.

[4] V. Yatsuk, T. Bubela, Y. Pokhodylo, Y. Yatsuk, R. Kochan, "Improvement of data acquisition systems for the measurement of physical-chemical environmental properties", in Proc. IEEE 9th Int. Conf. on "Intel.t Data Acq. and Adv. Comp. Syst.: Technology and Appl.", Bucharest, Romania, no.1, 2017, pp.41-46.

[5] O. Boyko, O. Chaban, "A mathematical model for optimizing the work of the primary care medical institution with taking into account the consumers opinion", in Proc. IEEE 13th Internat. Sc. and Techn. Conf. on Comp. Sc. and Inf. Techn., Lviv, 2018, pp. 103-107.

[6] M. Mykyychuk Metrological quality assurance at the production stage, Dr.Sc.Thesis, Lviv, Ukraine: Lviv Polytech. Nat. Un., 2012.

[7] M. Mesarovich, J. Takahara. General theory of systems: mathematical foundations. Moscow. RF: The World, 1978.

[8] V. Novoseltsev. Theoretical foundations of system analysis. Moscow. RF: Major, 2006.

[9] V. Dubovoy. Models of decision making in the management of distributed dynamic systems, Vinnytsya, Ukraine: Universum, 2008. 